\title{
ICOS sustains pathogenic T-cell survival in SLE mouse model
}

Inducible T cell co-stimulator (ICOS) supports follicular T-cell differentiation and is a known promoter of organ inflammation in mouse models of systemic lupus erythematosus (SLE). In a new report published in Immunity, ICOS ligand (ICOSL) expression by CD $11 c^{+}$ (also known as integrin $\alpha-\mathrm{X}$, encoded by Itgax) myeloid cells was associated with enhanced T-cell survival in the kidneys of lupus-prone mice.

\section{4 ...results suggest an autoantibody-independent role for ICOS in self-reactive T-cell survival in target organs... 77}

Expression of ICOS by T cells has been implicated in the disease process in lupus-prone mice, but the involvement of CD $11 c^{+}$dendritic cells (DCs), a major type of antigen-presenting cell (APC), in this process was unclear. In the study, lupus-prone MRL.Fas ${ }^{l p r}$ mice in which the gene encoding ICOSL (Icosl) was specifically deleted in $\mathrm{CD} 11 \mathrm{c}^{+}$cells (Itgax$\operatorname{Icos}^{\Delta}$ mice) had reduced proteinuria, interstitial nephritis and infiltrate size compared with Icosl-sufficient controls. Inflammation of the lung, but not of the skin, was also decreased in Itgax-Icosl ${ }^{\Delta}$ mice, but no substantial differences in T-cell accumulation in lymphoid tissues were detected between the two groups.

Previous reports have shown that complete Icos deletion in MRL.Fas ${ }^{l p r}$ mice leads to disease amelioration and reduced numbers of $\mathrm{T}$ extrafollicular helper $\left(\mathrm{T}_{\mathrm{EFH}}\right)$ cells, which express Bcl6, produce IL-21, and are thought to support autoantibody generation and provide B-cell help. In the present study, $\operatorname{Itgax}-I \cos l^{\Delta}$ mice had a $28 \%$ lower frequency of splenic $\mathrm{T}_{\mathrm{EFH}}$ cells among $\mathrm{CD}^{+} \mathrm{T}$ cells when compared with Icosl-sufficient controls. Unexpectedly, only a modest decrease in antinucleosome antibodies was observed in Itgax-Icos ${ }^{\Delta}$ mice, but, in agreement with the reduction in infiltrate size observed by histological analysis, the numbers of $\mathrm{CD} 4^{+}$and $\mathrm{CD} 8^{+}$ $\mathrm{T}$ cells in the kidneys of Itgax-Icosl ${ }^{\Delta}$ mice were reduced by $38.0 \%$ and $50.2 \%$, respectively. $\mathrm{T}_{\mathrm{EFH}}$ cells from Itgax-Icosl ${ }^{\Delta}$ mice showed normal expression of Bcl6 and IL-21, but had increase levels of apoptosis in kidney sections compared with control mice (8.8-fold increase in $\mathrm{TUNEL}^{+}$cells per $\mathrm{mm}^{2}$ ). This increase in apoptotic $\mathrm{T}_{\mathrm{EFH}}$ cells was associated with reduced phosphorylation and activity of the Akt survival pathway.

These results suggest an autoantibodyindependent role for ICOS in selfreactive T-cell survival in target organs of lupus-like disease. According to corresponding author Lino Teichmann, "the study represents a critical piece of the puzzle in understanding the relative roles of two major types of APCs-B cells and DCs/macrophages-in promoting systemic autoimmunity".

João H. Duarte

Original article Teichmann, L. L. et al. Local triggering of the ICOS coreceptor by $\mathrm{CD} 11 \mathrm{C}^{+}$myeloid cells drives organ inflammation in lupus. Immunity 42, 552-565 (2015) 